Original Research Article

\title{
Efficacy of oral L-arginine on amniotic fluid index in pregnant women with oligohydramnios attending antenatal clinic in a tertiary care hospital in Telangana, India
}

\author{
Md Amirunnisa Begum*, B. Krishna Sowmya, D. Shailendra, Y. Subbaratnam
}

Department of Pharmacology, Mediciti Institute of Medical Sciences, Ghanpur, Medchal, Telangana, India

Received: 24 February 2019 Accepted: 02 March 2019

*Correspondence to: Dr. Md Amirunnisa Begum, Email: mohammadamirunnisa@ gmail.com

Copyright: () the author(s), publisher and licensee Medip Academy. This is an openaccess article distributed under the terms of the Creative Commons Attribution NonCommercial License, which permits unrestricted noncommercial use, distribution, and reproduction in any medium, provided the original work is properly cited.

\begin{abstract}
Background: Oligohydramnios leads to feto-maternal morbidity and mortality. Though there is no specific treatment for oligohydramnios, use of L-arginine seems to be promising. As a nitric oxide donor, it causes vasodilatation, increases placental perfusion and finally increases amniotic fluid. However, data on the use of L-arginine for oligohydramnios is scarce. Hence, this study was aimed to evaluate the efficacy of oral L-arginine on Amniotic Fluid Index (AFI) and to document the pregnancy outcomes in women with oligohydramnios.

Methods: This was a prospective observational study conducted on pregnant women attending antenatal clinic (ANC) at Mediciti Institute of Medical Sciences (MIMS), Ghanpur, Telangana, India from $1^{\text {st }}$ January 2018 to $30^{\text {th }}$ June 2018.

Results: A total of 50 participants were enrolled and 4 participants among them were lost to follow- up. Mean age (SD) of the women enrolled was 23.3 (3.49) years. Mean gestational age (SD) at the time of diagnosis was 34.61 (1.53) weeks. Mean AFI (SD) at the time of diagnosis and after treatment with L-arginine were $6.8(1.3) \mathrm{cm}$ and $9.4(2.82) \mathrm{cm}$ respectively. After a mean treatment duration (SD) of 3.23 (1.38) weeks, a mean (SD) increase of AFI by $2.6(1.57) \mathrm{cm}(\mathrm{P}<0.0001)$ was observed. An increase of AFI was noted in $84.78 \%$ of cases $(\mathrm{P}<0.0001)$. Mean (SD) Gestational age at the time of delivery was 38.25 (1.48) weeks. Only $37 \%$ of participants required operational deliveries. Mean (SD) birth weight of the new borns was $2.54(0.47) \mathrm{kg}$. Neonatal Intensive Care Unit (NICU) admissions were required in $32.6 \%$ of new borns.

Conclusions: L-arginine is efficacious in improving AFI in oligohydramnios. AFI improvement could possibly lead to better neonatal outcomes by reducing preterm deliveries and operative interventions.
\end{abstract}

Keywords: Amniotic fluid index (AFI), L-arginine, Pregnancy, Oligohydramnios

\section{INTRODUCTION}

The fetus is surrounded by the amniotic fluid which serves as a cushion. ${ }^{1,2}$ The amniotic fluid is a vital liquid which contains nutrients and growth factors that are required for normal fetal growth and allows the assessment of fetal maturity. ${ }^{3}$ About $3-5 \%$ of pregnancies are affected by oligohydramnios, a condition with decreased amount of amniotic fluid, which results in placental insufficiency, impaired lung development in fetus and fetal growth restriction (FGR). ${ }^{1,4}$ Long-term complications of oligohydramnios include cord compression and increased chances of operative deliveries. ${ }^{1,4}$

The amniotic fluid volume is calculated with the fourquadrant technique done by ultrasound. ${ }^{1,4}$ It is the sum of the maximum vertical fluid pockets (measured in $\mathrm{cm}$ ) in each of the four quadrants. Quantitatively oligohydramnios is described as amniotic fluid index (AFI) $<5$ $\mathrm{cm}$ or $<5^{\text {th }}$ percentile for the gestational age or $5-10 \mathrm{~cm}$ as 
borderline or single deepest pocket (SDP) $<2 \mathrm{~cm}^{5-13}$ Though there is no specific treatment for oligohydramanios, use of L-arginine seems to be promising.

L-arginine, a semi-essential amino acid, is an endogenous precursor of nitric oxide (NO). It plays a vital role in regulation of blood flow in vascular beds and placental perfusion, vasodilatation and platelet aggregation. ${ }^{1}$

Because of these effects, it increases the volume and viscosity of blood in the feto-maternal circulation and promotes the intrauterine growth of the fetus (Figure 1). ${ }^{1}$

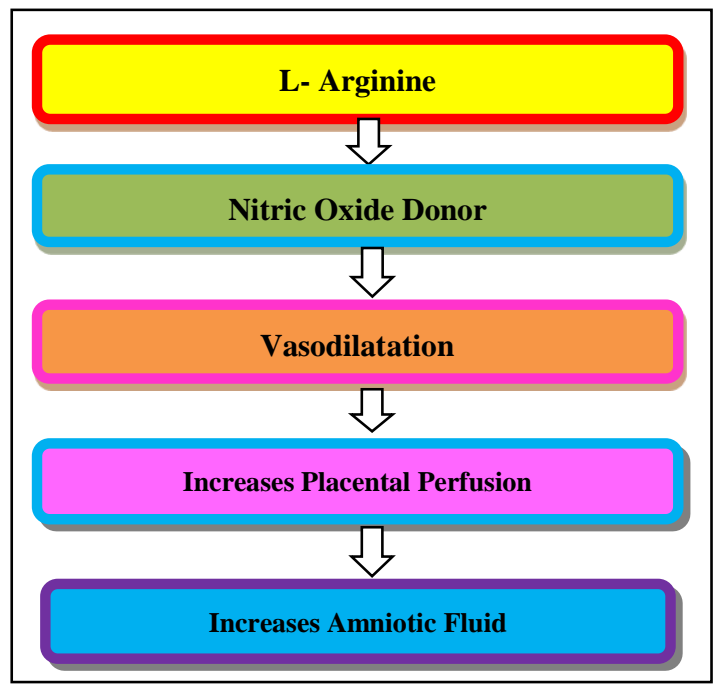

Figure 1: Mechanism of action of L-arginine.

As the literature on effect of L-arginine on amniotic fluid index is scarce, therefore, this study was conducted in pregnant women with oligohydramnios attending antenatal clinic (ANC) in a tertiary care hospital in Telangana, India with the objectives, to evaluate the efficacy of oral L-arginine on Amniotic Fluid Index (AFI) and to document the pregnancy outcomes.

\section{METHODS}

This was a prospective observational study conducted on pregnant women with oligohydramnios who attended antenatal clinic (ANC) at Mediciti Institute of Medical Sciences (MIMS), Medchal, Telangana, India from $1^{\text {st }}$ January 2018 to $30^{\text {th }}$ June 2018.

Ethics committee clearance was obtained from the Institutional ethics committee. Pregnant women who met inclusion criteria were enrolled after taking informed consent.

The literature showed that AFI of $8 \mathrm{~cm}$ was associated with an increased incidence of meconium staining, caesarean delivery, fetal distress, APGAR 7 or less at one minute. ${ }^{3,14}$ Hence, a cut-off value of $8 \mathrm{~cm}$ was chosen as inclusion criteria for this study.
The inclusion Criteria includes pregnant women of gestational age 28-36 weeks, with oligohydramnios (AFI $\leq 8 \mathrm{~cm}$ ), who were prescribed L-arginine for its treatment and willing to consent.

The exclusion criteria include pregnant women not willing to consent for the study, diagnosed with major congenital anomalies and history of having received treatment for oligohydramnios. The data was collected as per the study proforma which comprised of participant details including age, obstetric formula, parity and gestational age.

Author also collected the information which were chief complaints, investigations done ((haemoglobin, random blood sugar), ultrasonography (USG) scan (AFI)), no. of L-arginine sachets prescribed per day, treatment duration, mode of delivery, neonatal outcomes (birth weight, APGAR scores, NICU admissions, meconium stained liquor).

Each participant was prescribed L-arginine sachet containing $3 \mathrm{gm}$ of the active ingredient, which had to be dissolved in $100 \mathrm{ml}$ of water and taken two times a day orally for periods varying between 1 to 4 weeks. Participants were followed as per the regular antenatal check-up protocol and the change in AFI was noted in the USG scan done before delivery.

\section{Statistical analysis}

The data collected was entered into Excel spreadsheet and statistical analysis was done by applying the descriptive statistics to generate frequencies, proportions and percentages. Z-Test and student t-test of significance were used as appropriate. All the statistical analyses were done using Social Science Statistics Calculators (www.socscistatistics.com).

\section{RESULTS}

A total of 50 participants were enrolled and 4 participants among them were lost to follow- up.

The mean age $(\mathrm{SD})$ of the women $(\mathrm{n}=46)$ enrolled was 23.3 (3.49) years. Among them, 26 (56.52\%) were primigravidae and $20(43.47 \%)$ were multigravida. The incidence of oligohydramnios was more in primigravidae, which was statistically significant ( $\mathrm{P}<0.0001)$. Nulliparous women accounted to $67.39 \%$ of the participants whereas, multiparous women were $32.61 \%$. There was increased incidence of oligohydramnios in nulliparous women, which was statistically significant $(\mathrm{P}$ $<0.0001)$. All the details of participants regarding age wise distribution, gravidity and parity are depicted in Table 1.

The mean gestational age (GA) (SD) at the time of diagnosis was 34.61 (1.53) weeks. Among them, 10 participants were between 31-34 weeks of gestation and 26 participants were between 34-36 weeks of gestation. This 
data regarding distribution of women according to the gestational age is depicted in Table 2.

Table 1: Basic characteristics.

\begin{tabular}{|c|c|c|c|c|}
\hline Character & & No. of women (n) & Percentage $(\%)$ & P-value \\
\hline \multirow{3}{*}{ Age } & $<20$ & 4 & 8.69 & \\
\hline & $20-25$ & 30 & 65.21 & \\
\hline & $>25$ & 12 & 26.08 & \\
\hline \multirow{2}{*}{ Gravidity } & Primigravidae & 26 & 56.52 & \multirow{2}{*}{$<0.0001 *$} \\
\hline & Multigravidae & 20 & 43.47 & \\
\hline \multirow{2}{*}{ Parity } & Nulliparous & 31 & 67.39 & \multirow{2}{*}{$<0.0001 *$} \\
\hline & Multiparous & 15 & 32.61 & \\
\hline \multicolumn{2}{|c|}{ Total no. of participants } & 46 & & \\
\hline
\end{tabular}

*Z-Test, $\mathrm{P}<0.05$ is considered as statistically significant.

Table 2: Distribution of women according to gestational age (in weeks) at the time of diagnosis.

\begin{tabular}{|llll|}
\hline $\begin{array}{lll}\text { Gestational } \\
\text { age (in weeks) }\end{array}$ & $\begin{array}{l}\text { No. of } \\
\text { women (n) } \\
(\%)\end{array}$ & $\begin{array}{l}\text { Mean } \\
\text { gestational age } \\
\text { (in weeks) }\end{array}$ & SD \\
\cline { 1 - 2 } $28-31$ & $0(0)$ & & \\
\cline { 1 - 2 } $31-34$ & $10(21.74)$ & & 1.53 \\
\hline $34-36$ & $26(78.26)$ & & \\
\hline Total & 46 & & \\
\hline
\end{tabular}

Mean AFI (SD) at the time of diagnosis and after treatment with L-arginine were $6.8(1.3) \mathrm{cm}$ and $9.4(2.82) \mathrm{cm}$ respectively (Figure 2).

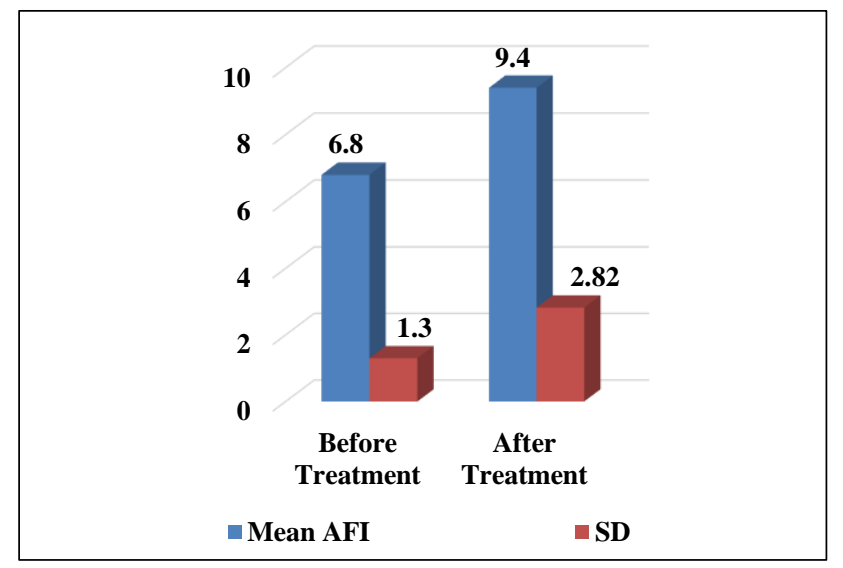

Figure 2: Improvement in AFI with L-arginine.

After a mean treatment duration (SD) of 3.23 (1.38) weeks, a mean (SD) increase of AFI by $2.6(1.57) \mathrm{cm}$ was observed, which was statistically significant $(\mathrm{P}<0.0001)$. A statistically significant increase of AFI was noted in $84.78 \%$ of cases $(\mathrm{P}<0.0001)$. No change in AFI was seen in $6.52 \%$ of cases and a decrease of AFI was seen in $8.7 \%$ of cases. Mean (SD) gestational age at the time of delivery was 38.25 (1.48) weeks. Only $17(36.95 \%)$ participants required operational deliveries whereas, 29 (63.04\%) participants delivered by normal vaginal delivery. The details regarding mode of delivery is depicted in Table 3.

Table 3: Mode of delivery.

\begin{tabular}{|ll|l|}
\hline Mode of delivery & Frequency (n) & Percentage (\%) \\
\hline Normal vaginal & 29 & 63.04 \\
\hline Caesarean section & 17 & 36.96 \\
\hline
\end{tabular}

The participants gave birth to a total of 46 (100\%) live neonates. There were no stillbirths or intrauterine deaths. Mean (SD) birth weight of the new borns was $2.54(0.47)$ $\mathrm{kg}$. Mean APGAR score (SD) at 1 minute and 5 minutes were $8(0)$ and $9(0)$ respectively. Meconium stained liquor was noticed only in $5(10.87 \%)$ deliveries. The data regarding the neonatal outcomes is depicted in Table 4.

Table 4: Neonatal outcomes.

\begin{tabular}{|lll|}
\hline Characteristics & \multicolumn{1}{l|}{ Value } \\
\hline Live births (n) & 46 \\
\hline Still births/IUDs \# (n) & 0 \\
\hline Mean birth weight (SD) in kg & $2.54(0.47)$ \\
\hline \multirow{2}{*}{ Mean (SD) APGAR score } & At 1 min & $8(0)$ \\
\cline { 2 - 3 } & At 5 mins & $9(0)$ \\
\hline \multirow{2}{*}{ Meconium stained liquor (n) } & Present & $5(10.87 \%)$ \\
\cline { 2 - 3 } & Absent & $41(89.13 \%)$ \\
\hline \# Intrauterine deaths. & & \\
\hline
\end{tabular}

Out of 46 new borns, $19(41.3 \%)$ were low birth weight $(\mathrm{LBW})(<2.5 \mathrm{~kg})$ new borns and the rest $27(58.7 \%)$ weighed $\geq 2.5 \mathrm{~kg}$. Mean AFI (SD) of women who gave birth to babies weighing $<2.5 \mathrm{~kg}$ and $\geq 2.5 \mathrm{~kg}$ was 8.74 $(2.75) \mathrm{cm}$ and $9.87(2.83) \mathrm{cm}$ respectively. The difference in AFI was not statistically significant $(\mathrm{P}=0.0915)$. Among 46 new borns, $15(32.6 \%)$ required Neonatal Intensive Care Unit (NICU) admission whereas, 31 $(67.4 \%)$ did not require NICU admission. The mean AFI (SD) of women whose babies required NICU admission was $8.57(3.18) \mathrm{cm}$ and who did not require NICU admission was $9.81(2.59) \mathrm{cm}$. The difference in AFI was 
not statistically significant $(\mathrm{P}=0.0826)$. The details regarding the mean AFI of the women in relation to the neonatal characteristics (birth weight and NICU admissions) are depicted in Table 5.

Table 5: AFI of the participants in relation to the neonatal characteristics.

\begin{tabular}{|lllll|}
\hline Characteristics & & Number $\mathbf{n}(\%)$ & Mean AFI (SD) $(\mathbf{c m})$ & P-value \\
\hline \multirow{2}{*}{ Weight of the baby } & $<2.5$ & $19(41.3)$ & $8.74(2.75)$ & $0.0915^{*}$ \\
\cline { 2 - 5 } & $\geq 2.5$ & $27(58.7)$ & $9.87(2.83)$ & $0.0826^{*}$ \\
\hline \multirow{2}{*}{ NICU admission } & Required & $15(32.6)$ & $8.57(3.18)$ & $9.81(2.59)$ \\
\cline { 2 - 4 } & Not required & $31(67.4)$ & & \\
\hline
\end{tabular}

*Student t-test, $\mathrm{P}<0.05$ is considered as statistically significant.

\section{DISCUSSION}

With the advent of ultrasonography, oligohydramnios is easily detectable. The invasive treatment options like amnioinfusion not only had variable success rates but also can lead to fetal loss. L-arginine is thus a promising alternative as it has diverse physiological functions such as improving amniotic fluid index, promoting intrauterine growth of the fetus, prolonging period of gestation and decreasing operative deliveries. ${ }^{1}$

Mean age (SD) of the women enrolled in this study was 23.3 (3.49) years which was similar to the study done in Karimnagar, Telangana and Wardha, Maharashtra, India. ${ }^{3,15}$

Majority of the cases were primigravidae, a similar finding to that of a study conducted in Karimnagar, Telangana and Udaipur, Rajasthan, India.,

In this study, the mean gestational age (SD) at the time of diagnosis was 34.61 (1.53) weeks whereas mean GA was $31.1 \pm 2$ weeks and 32.3 weeks in a study done in Wardha, Maharashtra and Powai, Maharashtra, India respectively. ${ }^{1,15}$

Mean AFI (SD) before and after treatment with L-arginine were noted to be $6.8(1.3) \mathrm{cm}$ and $9.4(2.82) \mathrm{cm}$ in this study, which is comparable with the values in the studies done at Powai, Maharashtra and Udaipur, Rajasthan, India. ${ }^{1,4}$

A mean (SD) increase of AFI by 2.6 (1.57) $\mathrm{cm}$ was observed in this study which is similar to that reported in the study done in Powai, Maharashtra and slightly more than that in Wardha, Maharashtra, India. ${ }^{1,15}$ However, the increase was statistically significant.

The duration of treatment with oral L-arginine varied from 2-4 weeks in this study. The mean treatment duration (SD) in this study was 3.23 (1.38) weeks, similar to that reported from a study conducted in Wardha, Maharashtra, India and slightly higher than that reported from another study conducted in Powai, Maharashtra, India. ${ }^{1,15}$ This difference can be explained by the difference in GA groups of the women enrolled. An increase of AFI was noted in a little more than four-fifths of the cases.

Only two-fifths of the participants required operational deliveries in this study as compared to three-fourths in a study conducted in Udaipur, Rajasthan and Powai, Maharashtra and four-fifths in a study conducted in Karimnagar, Telangana, India. ${ }^{1,3,4}$ Thus, operative deliveries in this study were far less than those in other studies.

Most common indication for operational deliveries was fetal distress apart from meconium stained liquor (MSL) and cord compression.

Meconium stained liquor was noticed in one-fifth of the deliveries in this study.

Low birth weight babies $(<2.5 \mathrm{~kg})$ accounted to two-fifths, in this study as compared to one fifth in a study conducted in Powai, Maharashtra and Udaipur, Rajasthan and one third in a study conducted in Karimnagar, Telangana, India. ${ }^{1,3,4}$ Hence, the incidence of LBW babies was slightly higher in this study than the other studies.

One-third of the new borns required NICU admissions in this study as compared to two fifths in a study conducted in Powai, Maharashtra and Udaipur, Rajasthan and one fifth in a study conducted in Karimnagar, Telangana, India. ${ }^{1,3,4}$ Therefore, the NICU admissions were slightly higher in this study than that in other studies.

Mean AFI in women giving birth to babies weighing $\geq 2.5$ $\mathrm{kg}$ was higher than that in those women giving birth to babies weighing $<2.5 \mathrm{~kg}$. This difference was not statistically significant. Similar findings were seen in the study done in Karimnagar, Telangana, India. ${ }^{3}$

Mean AFI in women whose babies did not require NICU admission was higher compared to those who required NICU admission. This difference was not statistically 
significant. Similar findings were seen in the study done at Karimnagar, Telangana, India. ${ }^{3}$

\section{CONCLUSION}

L-arginine is efficacious in improving AFI in oligohydramnios. By improving AFI it could possibly lead to better neonatal outcomes by reducing preterm deliveries and operative interventions.

Funding: No funding sources Conflict of interest: None declared

Ethical approval: The study was approved by the Institutional Ethics Committee

\section{REFERENCES}

1. Soni A, Garg S, Patel K, Patel Z. Role of L-arginine in oligohydramnios. J Obstetrics Gynecol Ind. 2016;66(1):279-83.

2. Mudaliar AL, Krishna Menon MK , Sarala Gopalan, Vanitha Jain, eds. Mudaliar and Menon's clinical obstetrics. 10th ed. Chennai; Orient longman pvt ltd; 2007 ;9788125028703.

3. Satyaprabha Siripurapu, Achanta Vivekanand, A Sarojini, Sethi Pruthwiraj. The role of amino acid infusion in isolated Oligohydramnios. Perspectives Med Res. 2015;3(1).

4. Sharma A, Dashora S, Sharma R, Chandra K, Gunawa MS. To study the effect of l-arginine in oligohydramnios to improve perinatal outcome. IOSR J Dental Med Sci (IOSR-JDMS). 2017;16(4):16-19.

5. Magann EF, Sandlin AT, Ounpraseuth ST. Amniotic fluid and the clinical relevance of the sonographically estimated amniotic fluid volume: oligohydramnios. J Ultrasound Med. 2011;30(11):1573-85.

6. Rutherford SE, Phelan JP, Smith CV, Jacobs NA. The four-quadrant assessment of amniotic fluid volume: an adjunct to antepartum fetal heart rate testing. Obstetrics Gynecol. 1987;70(3):353-6.

7. Baron C, Morgan MA, Garite TJ. The impact of amniotic fluid volume assessed intrapartum on perinatal outcome. Am J Obstetrics Gynecol. 1995;173(1):167-74.
8. Casey BM, McIntire DD, Bloom SL, Lucas MJ, Santos R, Twickler DM, et al. Pregnancy outcomes after antepartum diagnosis of oligohydramnios at or beyond 34 week's gestation. Am J Obstetrics Gynecol. 2000;182(4):909-12.

9. Moore TR, Cayle JE. The amniotic fluid index in normal human pregnancy. Am J Obstetrics Gynecol. 1990;162(5):1168-73.

10. Magann EF, Sanderson M, Martin JN, Chauhan S. The amniotic fluid index, single deepest pocket, and twodiameter pocket in normal human pregnancy. Am $\mathbf{J}$ Obstetrics Gynecol. 2000;182(6):1581-8.

11. Oosterhof H, Haak MC, Aarnoudse JG. Acute maternal rehydration increases the urine production rate in the near-term human fetus. Am J Obstetrics Gynecol. 2000;183(1):226-9.

12. Chamberlain PF, Manning FA, Morrison I, Harman CR, Lange IR. Ultrasound evaluation of amniotic fluid volume: I. The relationship of marginal and decreased amniotic fluid volumes to perinatal outcome. Am J Obstetrics Gynecol. 1984;150(3):245-9.

13. Morris JM, Thompson K, Smithey J, Gaffney G, Cooke I, Chamberlain $\mathrm{P}$, et al. The usefulness of ultrasound assessment of amniotic fluid in predicting adverse outcome in prolonged pregnancy: a prospective blinded observational study. BJOG: Inter J Obstetrics Gynaecol. 2003;110(11):989-94.

14. Jeng CJ, Lee JF, Wang KG, Yang YC, Lan CC. Decreased amniotic fluid index in term pregnancy. Clinical significance. J Reproductive Med. 1992;37(9):789-92.

15. Sreedharan R, Jajoo S. Effect of L-arginine on amniotic fluid index in oligohydramnios. Int $\mathrm{J}$ Reprod Contracept Obstet Gynecol. 2013;2:80-2.

Cite this article as: Begum MA, Sowmya BK, Shailendra D, Subbaratnam Y. Efficacy of oral Larginine on amniotic fluid index in pregnant women with oligohydramnios attending antenatal clinic in a tertiary care hospital in Telangana, India. Int J Basic Clin Pharmacol 2019;8:652-6. 